\title{
Management and Prevention of Diabetic Pressure Ulcers with Neuropathy
}

Suhair Shahwan*

Supervising Clinician, RMIT University, Health Science Department, Victoria, Australia

*Corresponding author: Suhair Shahwan, Supervising Clinician, RMIT University, Health Science Department, building 300 , University Hill Shopping Precinct 30 Janefield Drive, Bundoora VIC 3083, Australia, Tel: 61497229864; E-mail: sshahwan123@yahoo.com

Rec date: April 15, 2015 Acc date: July 23, 2015 Pub date: July 28, 2015

Copyright: () 2015 Shahwan S. This is an open-access article distributed under the terms of the Creative Commons Attribution License, which permits unrestricted use, distribution, and reproduction in any medium, provided the original author and source are credited.

\begin{abstract}
Diabetes has been the silent killer disease for many years and on the rise due to an increase in our body mass index. One of the most reported complications with diabetes is lower limb ulceration and amputation. The pathophysiology leading to ulceration is not fully understood. However ulceration has been associated with several risk factors which are neuropathy, ischaemia, foot musculoskeletal deformity resulting in an increase plantar pressure at the site of deformity. Patient with more than one risk factor is at a higher chance of developing foot ulcers and for this reason the patient should be assessed thoroughly in regards to their medical history when being evaluated by a specialist.
\end{abstract}

Keywords: Diabetes; Neuropathy; Ulceration; Pathophysiology

Diabetes affects the body on macro-vascular and microvascular level, due to its failure to deliver glucose adequately to the tissues of the body. Protecting the body from excess sugar in the blood is imperative in order to avoid coronary artery disease, peripheral artery disease, stroke (macro vascular level) and hypercholesterimia. Diabetes can also effect the body at the microvascular level which is pronounced through nephropathy, retinopathy and neuropathy [1-3].

Uncontrolled diabetes has also been linked to other conditions in the lower extremities such as Charcot neuropathic arthropathy. This condition is referred to as a structural deformity of the foot due to neuropathy that effect the bone, soft tissue and joints or trauma associated with long onset of diabetes. Treating Charcot foot with diabetes is still challenging and pressure ulcers commonly occur with this condition if the foot is not off loaded properly. Surgery might be another alternative that is also considered when conservative offloading methods fail. However, we should always bare in mind that diabetic patients with charcot foot should be monitored very closely even after successful offloading of high pressure areas of the foot. Patients should also check their sugar level on regular basis and keep up with their frequent visit to their podiatrist for any possible exacerbation of their neuropathic condition [3].

Prevention of foot ulcers with diabetic patients is important in reducing the amputation rate among this especial population. It has been estimated that approximately 40,000 amputations are performed yearly on patients with diabetes in the United States [4]. Wound ulcers, particularly in the foot region can occur at several locations and this is usually what identify the ulcer if it is neuropathic, pressure or ischaemic ulcer. In addition to other clinical findings associated with each type of ulcers upon examination. Extrinsic factors such as type of shoe they wear, non-compliant patient and inability of the patient to care for themselves, should also be considered when evaluating a wound. In general clinical practice guidelines for prediction and prevention of pressure ulcers should always be addressed in every community involved in treating lower extremity wounds [5].
In this review a critical appraisal of diabetic neuropathy as a diabetic complication factor and how it is related to altering peak pressure status of the diabetic foot will be discussed. In addition, best management procedures for this type of cases will also be addressed.

Neuropathic ulcers are ulcers usually as a result of loss of protective sensation in the feet which makes the patient's feet vulnerable to physical, chemical and thermal trauma. Pressure ulcers are usually interrelated with neuropathic ulcers because they are associated with motor neuropathy that is greatly related to feet deformities such as bunions, claw toe, mallet toe and other mid and rear foot deformity. This could lead to bony prominence areas as a result of abnormal joint deviations which as a result leads to an increase in plantar peak pressure in that region [5].

Common features with neuropathic ulcer as mentioned earlier, is a loss in sensation around the ulcer with callus deposit around the wound region. The wound tissue itself will appear pink in colour surrounded by callus. Foot feels warm upon touch and the physician usually could feel the pulses of the feet. These types of ulcers appear in the weight bearing areas of the foot such as the heel or over the dorsal aspect of the hammer toes and the plantar aspect of the metatarsal head. Other features associated with neuropathic ulcer is dry skin and fissures which is due to autonomic neuropathy [5].

Plantar ulcerations occur in the areas where there is a complete loss of protective sensation occurring as a result of an interaction between mechanical factors and abnormal connective-tissue properties in the neuropathic diabetic foot. The main mechanical factor that can trigger plantar ulceration of the foot, in conjunction with sensory impairment, is repetitive mechanical stress [6].

Pressure, as a risk factor in the repetitive mechanical stress, has been related to impaired mobility or impaired sensory perception (propioception). Delbridge et al. study was the first to question why some people with diabetic neuropathy develop plantar ulcers while others do not. Some studies documented that it was related to restricted joint mobility of the subtalar joint range of motion in neuropathic feet of adults with diabetes and that interfered with the foot's ability to absorb shock and transfer forces efficiently during gait [7]. 
Other studies thought the build of callus on the plantar aspect of the foot plays a crucial role in neuropathic ulceration. According to Young et al. studies, it was observed that plantar callus increases plantar pressure by behaving as a foreign body and it increases pressure in that region [8].

High peak plantar pressure and plantar callus as two major factors involved in the diabetic foot have encouraged many researchers to evaluate interventions in decreasing or preventing these factors. As a result several cushioning materials and thermoplastic orthoses have been successfully studied in the reduction of plantar pressure and callus in adults with diabetes $[9,10]$.

According to Duffin et al. study, it was found that there was no increase in plantar callus and high peak plantar pressure with diabetes compared to the non-diabetic patients group. Other studies such as Bevans and Bowker studies showed that plantar callus was just as common in development with diabetes and non-diabetic patients. However these risk factors could be devastating more with diabetic patients particularly if they are neuropathic. ill-fitting footwear as an extrinsic factor can have a crucial effect on the neuropathic diabetic foot and should be put into consideration as soon as possible [6].

High peak pressure was not a major factor in ulceration in Duffin et al. studies [6]. However the only noticeable difference between those with and without high peak plantar pressure was in pressure- time integral, which demonstrated statistical significance only in the group with diabetes. The intragroup comparison of diabetic subjects with and without plantar callus again showed similarities with the corresponding non-diabetic controls. Diabetic subjects with plantar callus were highly associated with older patients and who had a longer duration of diabetes, higher peak plantar pressure, higher pressuretime integral, and longer contact time than those without callus [6].

Further investigation is needed for the relationships between plantar callus, high peak plantar pressure, and other complications of diabetes, such as restricted joint mobility and neuropathy. This may be more clear as a better indication on why these abnormalities were noticed only in the group with diabetes.

Other studies by McPoil et al. which was conducted on American indians had different outcomes. The main purpose of this study was to demonstrate the magnitude and the time period of plantar pressures effecting the feet of American Indians with diabetes mellitus. The others purpose of this study was to find out if there is any differences in the range of motion of the ankle joint and first metatarsophalangeal joint existed between American Indians with and without diabetes [4].

This study excluded diabetic patients with history of present ulcers or history of previous ambulation or was not able to ambulate on their own. There were two groups divided into a group of diabetic patients with neuropathy (DWN) and a group of diabetic patients with no neuropathy (DNN). The diabetic neuropathic group were diagnosed by inability to sense a Semmes- Weinstein 5.07 monofilament on the plantar surface of the foot and reduced or absent ankle reflexes. The third group was a control group and consisted of 20 Pima Indians which consisted of four men and sixteen women without a history of diabetes and they were used for the possible influences of age or body weight. Gait analysis for all phases was done through an EMED-SF floor-mounted capacitance transducer matrix platform. Pressure data were gathered for five consecutive steps for both the left and the right foot (a total of ten steps).
Measurements of ankle dorsiflexion and metatarsophalangeal joint was done in a prone position. Diabetic neuropathic group had the highest value which was $616 \mathrm{kPa}$. The maximum pressure values for the other groups (control and DNN groups) were 533 and $518 \mathrm{kPa}$ respectively. The maximum peak pressure was identified in the central forefoot region for all three groups, and the next highest region of maximum peak pressure which also showed results similarities in pressure - time integral for all three groups was the hallux region, medial, and lateral forefoot during stance phase. The maximum peak pressures recorded for American Indian diabetic patients in the current study were much lower than peak pressure reported with other ethnicities in other studies such as Frykberg et al. study [11]. One reason for the discrepancy in the values for maximum plantar pressures between these previous studies and the current investigation was the use of different technologies to collect the pressure data. A second purpose of the study was to determine whether American Indians with diabetes showed decreased ankle and first metatarsophalangeal joint extension in comparison with non-diabetic American Indians [4].

For ankle dorsiflexion range of motion, the control group was remarkably different from the DNN and DWN groups but there was no difference documented between the mean values for the DNN and DWN groups. The mean ankle dorsiflexion range-of-motion values in the current study were higher for all groups than those previously reported by other studies such as Mueller et al. [12]. This final statement was related to the fact that the control and diabetic subjects in the Mueller et al. study were older in age. However, the duration of the diagnosis with diabetes mellitus was quite similar. Although, Mueller et al. did not indicate the ethnic background of the subjects used in their study, it appears that collagen-tissue changes associated with diabetes did not cause the same degree of reduction in ankle range of motion for American Indian diabetic patients evaluated in the current study [12].

The first metatarsophalangeal joint extension range of motion was significantly less for the DWN group than for the control and DNN groups [4]. In the present study, no significant differences were found between the control and DNN groups for first metatarsophalangeal joint extension range of motion values. For both ankle dorsiflexion and first metatarsophalangeal joint extension, the greatest decrease in range of motion values occurred with the DWN group, which also had the longest duration since diagnosis of diabetes mellitus year. These findings are in agreement with other published studies that have also reported decreased range of motion as a result of a reduction in collagen-tissue mobility associated with diabetes mellitus such as Armstrong et al. and Cavanagh et al. [13-14].

In addition to the previous findings in regards to plantar peak pressure studies and other factors eliciting the development of neuropathic pressure ulcer, it was documented by Cavanagh and Ulbrecht et al. and other few studies that in order for plantar ulceration to occur it has to exceed a value of $500 \mathrm{kPa}$ or above. However more investigation is needed in future studies to determine that this is the accurate value, since few studies were produced in that area.

Even though there was some limitation to Mcpoil et al. study due to non-aged match groups, the candidates participated in this study which were American Indians has been announced to have the highest rates of diabetes mellitus type two in the world. Another limitation factor to this study was not controlling the speed of the participants when they were asked to walk over the pressure platform. Other 
Page 3 of 3

studies indicated the prescribed speed for candidates during gait analysis so it will not alter normal gait pattern [4].

In general all these studies show a strong correlation between peak plantar pressure location and the anatomical region involved in the forefoot. In addition to the increase in the time integral pressure with diabetic patients with neuropathy in comparison with diabetic patients with no neuropathy. Another studies by kelly et al. also agreed with our previous studies results, in regards to the fact that peak plantar pressure was mostly increased in the forefoot region during late stance phase in comparison to other phases. Kelly's et al. study was done to determine how to enhance the footwear design in order to decrease the forces acting on the foot during weight bearing. The only limitation to that study was the difference in the neutral position considered for orthotic devices during molding and that was done by taking an impression of the foot while the patient is sitting with the knee flexed to 90 degree or while standing [10].

This neutral position was also not identical to the position of the foot at the time of peak plantar pressure during walking which was another limiting factor. Another limitation to this study was conducting it on patients with forefoot deformities and with metatarsal amputation which could naturally alter the gait and peak plantar pressure.

In general according to kelly et al. study, the best footwear design to reduce the peak force during the late stance phase is a shoe that focus on increasing the weight bearing surface area during the late stance phase. This will minimise the trauma to the neuropathic foot while weight bearing. Other studies by Fernandez et al. revealed a decrease in reulceration of diabetic patients with the use of custom made orthotics and insoles. Despite the fact there was no control group, the candidates of this study all had a history of recurrent ulceration due to increase in peak plantar pressure when ambulating.

Fernandez et al. revealed the possibility of reducing peak plantar pressure through the use of complete orthotic treatment. During gait it was documented that plantar pressure increases in the forefoot during late stance and active propulsion stage across a smaller area as the support base of the foot was decreasing. This study agrees with the previous study, Duffin, A. et al. study in regards to the fact that peak plantar pressure of the forefoot during gait is decreased by $20 \%$ in peak plantar pressure and pressure time integral when using a $3-\mathrm{mm}$ flat cushioning in the group of diabetes with high plantar pressure and contact time. Peak Plantar pressure was decreased even more with custom made orthotics $[6,9]$.

A combination of both modalities reduced the peak plantar pressure even more than the value obtained with custom made orthotics and $3 \mathrm{~mm}$ PPT flat cushioning alone. On the other hand contact time factor was increased only with custom-made orthoses and decreased with PPT cushioning and a combination of both modalities decreased the contact time but it was still higher in value with contact time in relationship to using the PPT cushioning modality alone. Pressure time integral was not indicated with kelly et al. study which makes us believe there should be more investigation in regards to pressure time integral. Duffin et al. study also excluded any participants with forefoot deformity and previous amputation [6].

Snyder et al. studies of the best management of diabetic foot ulcers through optimal off-loading, showed that there was an increase in healing of plantar pressure ulcers with off-loading adherence. It also indicated that the best methods of offloading a pressure ulcer is by using the total contact cast or a non-removable cast walkers. In addition to the fact that off-loading method is cost-effective. This study was based on literature search through the PubMed for best offloading methods and using a Grading system for recommendation, assessment, development and evaluation for the quality of evidence that support the type of off-loading device [10].

In conclusion it has been documented that custom made orthotics and accommodative orthotics help in preventing ulceration with diabetic patients with neuropathy and with a history of recurrent ulceration. This shows through the decrease in the peak plantar pressure and pressure integral time that is highly increased at the plantar aspect of the medial, central, lateral forefoot and the plantar aspect the hallux. Total contact cast was the gold standard to off-load a current pressure ulcer. However there should be more studies that emphasise on neuropathic diabetic shoe design in correlation to peak plantar pressure, pressure time integral and phase with the highest peak pressure during gait analysis.

\section{References}

1. Sage, Ronald A, Webster JK, Fisher SG (2001) 'Outpatient Care And Morbidity Reduction In Diabetic Foot Ulcers Associated With Chronic Pressure Callus'. Journal of the American Podiatric Medical Association 91: 275-279.

2. Westphal C, Neame IM, Harrison JC, Bower VM, Gurr JM (2011) A diabetic foot ulcer pilot study: does silicone gel sheeting reduce the incidence of reulceration? J Am Podiatr Med Assoc 101: 116-123.

3. Fabrin J, Larsen K, Holstein PE (2000) Long-term follow-up in diabetic Charcot feet with spontaneous onset. Diabetes Care 23: 796-800.

4. McPoil TG, Yamada W, Smith W, Cornwall M (2001) The distribution of plantar pressures in American Indians with diabetes mellitus. J Am Podiatr Med Assoc 91: 280-287.

5. Frykberg RG (1997) Team approach toward lower extremity amputation prevention in diabetes. J Am Podiatr Med Assoc 87: 305-312.

6. Duffin AC, Kidd R, Chan A, Donaghue KC (2003) High plantar pressure and callus in diabetic adolescents. Incidence and treatment. J Am Podiatr Med Assoc 93: 214-220.

7. Delbridge L, Perry P, Marr S, Arnold N, Yue DK, et al. (1988) Limited joint mobility in the diabetic foot: relationship to neuropathic ulceration. Diabet Med 5: 333-337.

8. Young MJ, Cavanagh PR, Thomas G, Johnson MM, Murray H, et al. (1992) The effect of callus removal on dynamic plantar foot pressures in diabetic patients. Diabet Med 9: 55-57.

9. Fernandez ML, Lozano RM, Diaz MI, Jurado MA, Hernandez DM, et al. (2013) How effective is orthotic treatment in patients with recurrent diabetic foot ulcers? J Am Podiatr Med Assoc 103: 281-290.

10. Snyder RJ, Frykberg RG, Rogers LC, Applewhite AJ, Bell D, et al. (2014) The management of diabetic foot ulcers through optimal off-loading: building consensus guidelines and practical recommendations to improve outcomes. J Am Podiatr Med Assoc 104: 555-567.

11. Frykberg RG, Lavery LA, Pham H, Harvey C, Harkless L, et al. (1998) Role of neuropathy and high foot pressures in diabetic foot ulceration. Diabetes Care 21: 1714-1719.

12. Mueller MJ, Diamond JE, Delitto A, Sinacore DR (1989) Insensitivity, limited joint mobility, and plantar ulcers in patients with diabetes mellitus. Phys Ther 69: 453-459.

13. Armstrong DG, Peters EJ, Athanasiou KA, Lavery LA (1998) Is there a critical level of plantar foot pressure to identify patients at risk for neuropathic foot ulceration? J Foot Ankle Surg 37: 303-307.

14. Cavanagh PR, Ulbrecht JS (1994) 'Clinical Plantar Pressure Measurement In Diabetes: Rationale And Methodology'. The Foot 4: 123-135. 